\title{
Antibody Response to Mannheimia Haemolytica Leukotoxin in Cattle with Respiratory Tract Disease
}

\author{
S. Cavirani*, ${ }^{*}$, S. Taddei ${ }^{1}$, C.S. Cabassi ${ }^{1}$, F. Ghidini ${ }^{1}$, C. Piancastelli ${ }^{1}$ and C.F. Flammini ${ }^{1}$ \\ ${ }^{I}$ Department of Animal Health, University of Parma, Via del Taglio, 8, 43100 Parma, Italy
}

\begin{abstract}
In order to obtain data concerning the involvement of Mannheimia haemolytica in bovine respiratory disease (BRD) outbreaks, a serological survey was carried out on paired (acute- convalescent) sera from 1310 beef and 810 dairy cattle collected in 262 BRD outbreaks in Italian herds during 2002-2006. No vaccination program to M. haemolytica A1 was applied in the investigated herds. For each outbreak, 5 to 12 animals were considered for serum sampling. An enzyme linked immunosorbent assay (ELISA) was used to determine serum antibody response to M. haemolytica leukotoxin (LKT). Seroconversion involved 467 animals (22\%), 314 beef cattle (24\%) and 153 dairy cattle (19\%), respectively. On a serological basis, $M$. haemolytica involvement was detected in $162(62 \%)$ BRD outbreaks. Prevalence of seroconversion ranged from $20 \%$ to $60 \%$. Concurrent seroconversion to $M$. haemolytica and the main bovine respiratory viruses was recorded in $141(54 \%)$ outbreaks. Seroconversion to M. haemolytica LKT involved mainly cattle not vaccinated to BRD viral agents.
\end{abstract}

Keywords: Bovine, Mannheimia haemolytica, leukotoxin, antibodies

\section{INTRODUCTION}

Shipping fever, or pneumonic pasteurellosis, is a major component of the bovine respiratory disease (BRD) complex. Mannheimia haemolytica is a common commensal organism of healthy cattle [1] which is implicated as a cause of fibrinous pneumonia in young calves (enzootic pneumonia), in weaned-beef animals (shipping fever) as well as in dairy cattle [2]. Fibrinous pneumonia attributed to $M$. haemolytica is the most important cause of BRD mortality. $M$. haemolytica serotype A1 is recognised worldwide as the predominant serotype involved in bovine pneumonia [1]. Following stress factors, M. haemolytica A1, that normally resides in the tonsillar crypt as part of the microbial flora of healthy cattle [1], replicates rapidly, colonizes the nasopharynx, and is carried to the lung via aerosolized droplets. During logarithmic growth phase, the organism secretes leukotoxin (LKT). The presence of a specific binding site for $M$. haemolytica LKT, located on bovine leukocytes, has been recently hypothesized to explain activation and lytic activity of $M$. haemolytica [3, 4]. M. haemolytica LKT also stimulates bovine alveolar macrophages to express procoagulant activity on their surface which can amplify fibrin deposition [5].

LKT elicits serum antibody responses in cattle after natural exposure to the microrganism [6] as well as following treatment with vaccines containing LKT [7]. Since the production of LKT is linked to active replication of M. haemolytica, detection of specific antibody response can be considered an indirect demonstration of its involvement in respiratory disease. Antibody response has been measured by labour-intensive cytotoxin (LKT) neutralization assay [8], and also by enzyme-linked immunosorbent assay (ELISA) [9]. In Italy, the involvement of $M$. haemolytica in BRD outbreaks

*Address correspondence to this author at the Department of Animal Health, University of Parma, Via del Taglio 8, 43100 Parma, Italy; Tel: +390521032667; Fax: +390521032672; E mail: sandro.cavirani@unipr.it has been demonstrated previously by isolation in cultures [10]. Since there is difficulty in obtaining relevant samples (nasal swabs and/or lung tissue) from acutely-affected animals, the role of $M$. haemolytica in inducing respiratory outbreaks in cattle might be underestimated. Therefore, serological investigations to detect seroconversion to M. haemolytica LKT could allow to better elucidate the prevalence of pneumonic pasteurellosis among BRD outbreaks.

In order to obtain data on the involvement of $M$. haemolytica in BRD outbreaks occurring in Italy, a serological survey was carried out on paired serum samples from beef and dairy cattle experiencing BRD outbreaks.

\section{MATERIALS AND METHODOLOGY}

\section{Animals}

Paired (acute-convalescent) serum samples were collected from 1310 beef and 810 dairy cattle in 262 BRD outbreaks which occurred in Italian herds during 2002-2006. Clinical diagnosis of BRD was mainly based on the following symptoms: nasal and ocular discharge, fever, coughing and labored breathing. No vaccination program for $M$. haemolytica A1 was applied in the examined herds.

\section{Serum Samples}

For each BRD outbreak, a number of animals, ranging from 5 to 12 , were serum sampled.

Sera were kept frozen at $-20^{\circ} \mathrm{C}$ prior to use. Those used in virus neutralization and hemagglutination tests were first heat inactivated at $56^{\circ} \mathrm{C}$ for $30 \mathrm{~min}$.

\section{Serology}

An indirect enzyme linked immunosorbent assay (ELISA) was used to determine serum antibody response to M. haemolytica LKT.

The antigen for ELISA was a purificated preparation of LKT obtained according to the method of Clinkenbeard et al. 
[11] with minor modifications. Briefly, the LKT was precipitated with $40-60 \%$ of ammonium sulfate from supernatant of 11 logarithmic-phase culture of $M$. haemolytica A1 reference strain ATCC BAA-409 grown in RPMI-1640 medium at $37^{\circ} \mathrm{C}$ in a shaking incubator. The precipitate was resuspended in $10 \mathrm{ml}$ of $3 \mathrm{M}$ guanidine, $50 \mathrm{mM}$ NaHPO4, 100 $\mathrm{mM} \mathrm{NaCl}$ and dialyzed against phosphate buffered saline (PBS). The presence of LKT was assessed by SDS-PAGE, using $4 \%$ stacking and $10 \%$ resolving gels. To reveal the molecular weight of obtained LKT, the gel was electrophoretically blotted onto Immobilon-P membrane (Millipore) according to the method of Towbin et al. [12]. After blotting, the membrane was blocked with $3 \%$ gelatin in tris-buffered saline (TBS) and probed with a monoclonal antibody (MAb) to M. haemolytica A1 LKT. MAb MM601 was kindly provided by Dr. S. Srikumaran (University of Nebraska, Lincoln, Nebraska, USA). After washing, the membrane was incubated with goat anti-mouse immunoglobulin G conjugated with horseradish-peroxidase (Sigma). The immunoblot was developed using the substrate diaminobenzidine (Sigma). Immunoblot analysis detected the presence of the LKT band at a molecular weight of approximately $100 \mathrm{kDa}$ (Fig. 1).

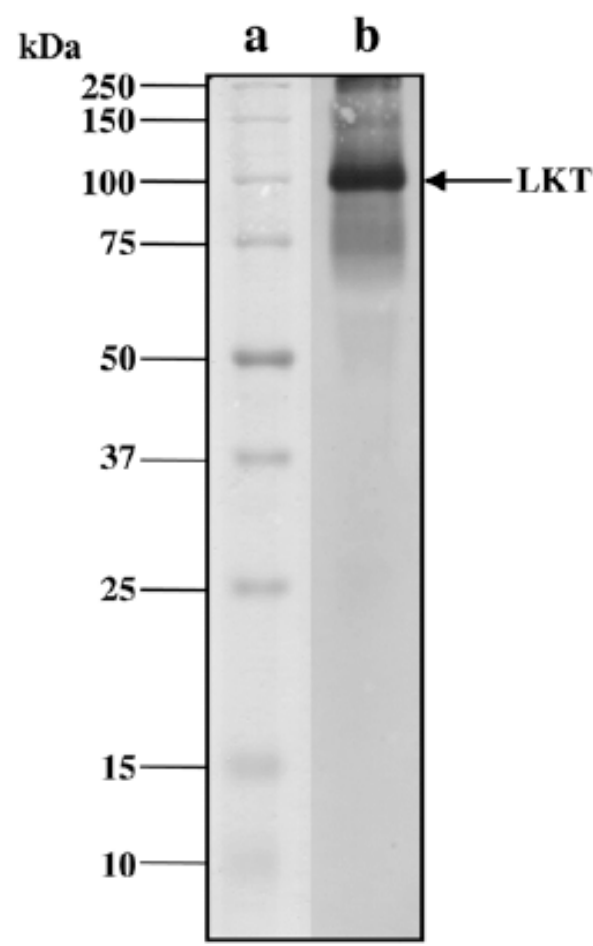

Fig. (1). Immunoblot analysis of M. haemolytica leukotoxin (LKT) preparation: (a) molecular weight markers, (b) immunoblot of $M$. haemolytica LKT preparation reacting with anti-LKT MAb MM601 revealing a band at approximately $100 \mathrm{kDa}$.

For ELISA, wells of 96 well microtitration plates were coated with $100 \mu \mathrm{l}(15 \mu \mathrm{g})$ of the LKT preparation per well. Paired serum samples were tested by an end-point assay, performing serial two-fold dilutions from 1:20 to 1:2560. The extent of antibody binding to LKT was detected by using a 1:200 dilution of horseradish peroxidase conjugate affinity purified rabbit anti bovine IgG. For colour development, a substrate containing phenylendiamine was added. Samples with a corrected optical density $(\mathrm{COD})>0.25$ were regarded as positive for antibody to LKT.
Infection with $M$. haemolytica was demonstrated by seroconversion (from negative to positive) or significant (4 fold) rise in antibody titer to LKT of paired acuteconvalescent serum samples. Involvement of $M$. haemolytica in a BRD outbreak was demonstrated by detection of one or more animals showing seroconversion to LKT.

In addition, all the paired serum samples were tested for antibodies to the main bovine respiratory viruses: Bovine Herpesvirus 1 (BHV-1), Bovine Viral Diarrhea Virus (BVDV) and Bovine Coronavirus (BC) by virus neuralization test (VN), Bovine Respiratory Syncytial Virus (BRSV) by indirect fluorescent antibody assay (IFA) and Parainfluenza 3 (PI3) by hemagglutination inhibition test (HI). In all the cases, seroconversion was demonstrated according to the criteria previously indicated.

\section{RESULTS}

Results concerning seropositivity to LKT of M. haemolytica in paired serum samples from cattle with respiratory disease are recorded in Table $\mathbf{1}$.

At the first sampling (acute serum) 66 out of 2120 cattle (3\%) showed antibody response to LKT. Seroconversion involved 467 animals (22\%) in 162 outbreaks (62\%), of which 314 beef cattle (24\%) in 94 outbreaks (69\%) and 153 dairy cattle (19\%) in 68 outbreaks (54\%). In the BRD outbreaks where seroconversion to $M$. haemolytica occurred were involved from $20 \%$ to $60 \%$ of the animals.

In addition, the seroconversion rate in beef cattle (24\% for the animals and $69 \%$ for the outbreaks) was significantly higher $(\mathrm{p}<0.01$ for the animals and $\mathrm{p}<0.05$ for the outbreaks) than in dairy cattle (19\% for the animals and $54 \%$ for the outbreaks).

In dairy cattle, seroconversion to LKT involved 153 animals, of which $112(73 \%)$ were calves and heifers and 41 $(27 \%)$ cows. In beef cattle, $224(71 \%)$ out of the 314 seroconverting animals were from BRD outbreaks occurring within two weeks from the arrival at the feedyard. Conversely, BRD episodes occurring in dairy cattle could not be linked to specific events or phases in the production cycle.

Concurrent seroconversion to M. haemolytica LKT and viruses was recorded in 305 animals (14\%) from 141 outbreaks $(54 \%)$. Viruses involved in concurrent seroconversion with $M$. haemolytica LKT were BRSV ( $\mathrm{n}=141)$, BVDV $(\mathrm{n} .=74), \mathrm{BC}(\mathrm{n} .=69), \mathrm{PI} 3(\mathrm{n} .=55)$ and BHV-1 (n.= 23). It was rather common $(\mathrm{n} .=57)$ to detect an antibody response towards M. haemolytica LKT and two or more respiratory viruses in the same animal. Mortality rate was $12 \%$ and $10 \%$ in the outbreaks showing concurrent seroconversion and LKT seroconversion alone, respectively.

On the basis of herd history concerning sanitary programs, it was recorded that $340(73 \%)$ out of 467 animals seroconverting to $M$. haemolytica LKT were unvaccinated against viral respiratory pathogens, BHV-1, BRSV, BVDV, PI3.

\section{DISCUSSION AND CONCLUSION}

This field study confirmed the involvement of M. haemolytica in bovine respiratory tract disease as has previously been reported for bacterial isolation data in BRD outbreaks occurring in cattle populations reared in the same area [10]. 
Table 1. Antibody Response to M. haemolytica Leukotoxin (LKT) in Serum Samples Collected from Cattle with Acute Respiratory Disease

\begin{tabular}{|c|c|c|}
\hline Animal category & Examined & Seroconversion* \\
\hline \hline Beef cattle & & $314(24 \%)$ \\
Animals & 1310 & $94(69 \%)$ \\
Outbreaks & 137 & $153(19 \%)$ \\
Dairy cattle & & $68(54 \%)$ \\
Animals & 810 & 125 \\
\hline
\end{tabular}

*Seroconversion (from negative to positive) or significant (4 fold) rise in antibody titer to LKT of paired acute-convalescent serum samples.

The immunological response to LKT measured here indicated that $M$. haemolytica was actively replicating for a time in most cattle in course of acute respiratory episodes. Antibody response (seroconversion) to LKT in paired (acuteconvalescent) serum samples indicates $M$. haemolytica as a pathogenic cause of the observed respiratory episodes. Since there is a short period of active colonization of M. haemolytica, which consequently limits the period for isolating bacteria by nasal swabbing, serological investigation could contribute to better diagnosis.

In our survey, seroconversion to M. haemolytica LKT was detected in $22 \%$ of the diseased cattle belonging to $62 \%$ of the BRD outbreaks where prevalence of individual seroconversion ranged from $20 \%$ to $60 \%$. The serological findings pointed out that the BRD outbreaks under investigation experienced $M$. haemolytica active infection. M. haemolytica should be considered as one of the main causes but not necessarily the primary cause of the disease, as our data indicate the high prevalence of concurrent seroconversion to $M$. haemolytica LKT and viruses.

As expected, BRSV showed the higher prevalence of seroconversion among the viruses involved in the examined BRD outbreaks. Surprisingly, a high prevalence of seroconverted animals was associated with BVDV and BC, which are most frequently identified as agents of enteric disease. However, both viruses have been involved in respiratory outbreaks occurred in Italy [13].

It is well known that BVDV causes a respiratory syndrome. BVDV replicates in the mucosal cells of the respiratory tract eliciting lung tissue damage [14]. In addition, immunosuppression following BVDV infection favours respiratory colonization with other viruses and bacteria, in particular M. haemolytica [15].

The role of $\mathrm{BC}$ in inducing $\mathrm{BRD}$ was investigated and ascribed to the existence of coronavirus strains showing tropism and pathogenic activity for the respiratory tract of cattle [16].

In our survey, the combination of $M$. haemolytica and viruses appears to have increased the pathogenic activity of the bacterium, since seroconversion to LKT involved mainly cattle that were not vaccinated to BRD viral agents. Vaccination to bovine respiratory viruses, BVDV included, should therefore contribute to the control of M. haemolytica infection as well. Consequently, the lack of a vaccination program to viruses has to be considered a risk factor for the development of $M$. haemolytica fibrinous pneumonia.
It has been shown that detection of an antibody response to LKT is useful in detecting an active infection status. Moreover, LKT antibodies can also prevent or reduce the occurrence of fibrinous pneumonia. Individual studies which have evaluated the efficacy of $M$. haemolytica virulence factors have claimed that a significant correlation exists between high antibody levels to LKT and an overall protection against intratracheal or transthoracic intrapulmonic challenge with live M. haemolytica [17]. Therefore, M. haemolytica A1 LKT is currently included in vaccine preparations that are available commercially in the cattle industry.

The observation of a high prevalence of concurrent infections involving $M$. haemolytica and viruses suggests that the routine treatment of beef and dairy cattle with combined vaccines for the prevention of viral infections and pasteurellosis would be beneficial. Experimental studies of these combinations have generally yielded favourable results [18].

In order to establish the best age and time for vaccination, the finding that in dairy cattle most of seroconversions were detected in calves is important. Vaccination of dams, which will improve specific passive immunity [19], appears to be a promising approach to reduce pneumonia occurrence in young animals.

For beef cattle, our evidence showing the occurrence of BRD after shipment, combined with the results of field trial carried out across a wide geographic range of locations, types and sources of the animals within different managements [20], suggests that vaccination administered upon arrival at the feedyard can reduce mortality due to respiratory disease.

\section{REFERENCES}

[1] Frank GH. Pasteurellosis of cattle. In: Pasteurella and pasteurellosis, Adlam C.F. and Rutter J.M. Ed. New York, Academic Press. 1989; 197-222.

[2] Whiteley LO, Maheswaran SK, Weiss DJ, Ames TR, Kannan MS. Pasteurella haemolytica A1 and bovine respiratory disease: pathogenesis. J Vet Intern Med 1992; 6: 11-22.

[3] Brown JF, Leite F, Czuprynski CJ. Binding of Pasteurella haemolytica leukotoxin to bovine leukocytes. Infect Immun 1997; 65: 3719-3724.

[4] Thumbikat P, Dileepan T, Kannan MS, Maheswaran SK. Characterization of Mannheimia (Pasteurella) haemolytica leukotoxin interaction with bovine alveolar macrophage beta2 integrins. Vet Res 2005; 36: 771-786.

[5] Car BD, Suyemoto M, Neilsen NR, Slauson DO. The role of leukocytes in the pathogenesis of fibrin deposition in bovine acute lung injury. Am J Pathol 1991; 138: 1191-1198.

[6] McBride JW, Corstvet RE, Paulsen DB, McClure JR, Enright FM Systemic and pulmonary antibody responses of calves to Pas- 
teurella haemolytica after intrapulmonary inoculation. Am J Vet Res 1992; 53: 1889-1894.

[7] Srinand S, Hsuan SL, Yoo HS, Maheswaran SK, Ames TR, Werdin RE. Comparative evaluation of antibodies induced by commercial Pasteurella haemolytica vaccines using solid phase immunoassays. Vet Microbiol 1996; 49: 181-195.

[8] Gentry MJ, Confer AW, Kreps JA. A simple visual assay for determination of Pasteurella haemolytica cytotoxin neutralizing antibody titers in cattle sera. J Clin Microbiol 1985; 22: 968-972.

[9] Mosier DA, Confer AW, Hall SM, Gentry MJ, Panciera RJ. Enzyme-linked Immunosorbent Assay for detection of serum antibodies to Pasteurella haemolytica cytotoxin (leukotoxin) in cattle. J Clin Microbiol 1986; 24: 218-222.

[10] Cavirani S, Candotti P, Guadagnini PF, Pozzi P, Allegri G. Pasteurella haemolytica as cause of bovine respiratory disease (BRD) in dairy and beef cattle in Italy: Proceedings of the 20th World Buiatrics Congress; 1998 Jul 6-10; Sydney, Australia. p. 1138.

[11] Clinkenbeard KD, Clarke CR, Hague CM, Clinkenbeard P, Srikumaran S, Morton RJ. Pasteurella haemolytica leukotoxininduced synthesis of eicosanoids by bovine neutrophils in vitro. $\mathbf{J}$ Leukoc Biol 1994; 56: 644-649.

[12] Towbin H, Staehelin T, Gordon J. Electrophoretic transfer of proteins from polyacrilamide gels to nitrocellulose sheets: procedure and some applications. Proc Natl Acad Sci USA 1979; 76: 43504354.
[13] Cavirani S, Allegri G, Donofrio G, Cabassi CS, Ceccato C, Ballottin M. Anticorpi verso Bovine Viral Diarrhea Virus (BVDV) in bovini da carne allevati in Nord Italia [in Italian]: Atti della Società Italiana di Buiatria; 1999 Jun 18-20; Marina di Ragusa, Ragusa, Italy. p. 303-309.

[14] Stott EJ, Thomas LH, Collins AP. A survey of virus infections of the respiratory tract of cattle and their association with disease. J Hyg (Lond) 1980; 85: 257-270.

[15] Brownlie J. The pathogenesis of bovine viral diarrhea virus infections. Rev Sci Tech 1990; 9: 43-59.

[16] Hasoksuz M, Lathrop S, Al-dubaib MA, Lewis P, Saif LJ. Antigenic variation among bovine enteric coronaviruses (BECV) and bovine respiratory coronaviruses (BRCV) detected using monoclonal antibodies. Arch Virol 1999; 144: 2441-2447.

[17] Gentry MJ, Confer AW, Panciera RJ. Serum neutralization of cytotoxin from Pasteurella haemolytica serotype 1 and resistance to experimental bovine pneumonic pasteurellosis. Vet Immunol Immunopathol 1985; 9: 239-250.

[18] Mosier DA, Confer AW, Panciera RJ. The evolution of vaccines for bovine pneumonic pasteurellosis. Res Vet Sci 1989; 47: 1-10.

[19] Hodgins DC, Shewen PE. Preparturient vaccination to enhance passive immunity to the capsular polysaccharide of Pasteurella haemolytica A1. Vet Immunol Immunopathol 1996; 50: 67-77.

[20] Pollreisz JH. Pooled analysis of pasteurella vaccination trial: Proceedings of the 19th World Buiatrics Congress; 1996 Jul 8-12; Edinburgh, United Kingdom. p. 13-15. 